\title{
The Simulation and Analysis of Uplink Antenna Arraying Power Combining Efficiency
}

\author{
Ke Sun ${ }^{1, a}$, Xiaomin $\mathrm{Hou}^{1, \mathrm{~b}}$ \\ ${ }^{1}$ Equipment Academy, Beijing 101416, China \\ ask2220060710@126.com, b13801244993@139.com
}

Keywords: Uplink antenna arraying, the loss of synthesis gain, the synthesis efficiency, the number of antennas, the phase error.

\begin{abstract}
In order to analyze the effect of uplink antenna arraying calibration synthesis, this paper explores the factors about the loss of antenna arraying combined gain from the theoretical analysis and simulation analysis on two levels. At first summary the foreign research about the experiments of uplink antenna array synthesis. Then describe the theoretical background about power combining and derive the relationship about power combining efficiency, the number of antennas and the phase error. Finally verify the quantitative relationship against the loss of power synthesis gains, the number of antennas and the phase error through simulation technology。 The results show that as long as the good control of the range on various factors. This conclusion has played a certain role in paving the way against the analysis of phase error for the antenna arraying.
\end{abstract}

\section{Introduction}

Since the spacecraft has a very far distance in deep space exploration, the deep space detector and the ground control station will face a huge path loss of signaling hundreds of millions of kilometers away. It will make very demanding performance requirements on ground control system. At this stage, the remote telemetry and communications tasks are completed by a single large-diameter antenna to in the deep space exploration. It is very difficult to continue to improve system performance by increasing the antenna aperture antenna. When the development of conventional antenna technology encountered a "bottleneck", the uplink antenna arraying emerged as a new concept. It has superior performance, reliable stability, low cost and many other advantages 0 [2]. The uplink arraying far-field consists of a plurality of aligned phase-precision antenna. The value of EIRP is $\mathrm{N}^{2} \mathrm{GP}$. Wherein, $\mathrm{N}$ is the number of antennas, $\mathrm{G}$ is the gain of a single antenna, $\mathrm{P}$ is the transmission power of antenna. The EIRP of uplink arraying are proportional to the square of the number of antennas. Thus, in theory, it can achieve higher values of EIRP than single large diameter antenna.

In recent years, many foreign research institutions, especially JPL has done a lot of research on key technologies of uplink arraying. At the same time take advantage of four $34 \mathrm{~m}$ antenna of Goldstone Deep Space Station to conduct several experiments. Next, the progress of these tests are introduced.

In February 25, 2006, V.A.Vilnrotter et al of JPL at the Goldstone Deep Space Communications Station conducted a demonstration test about uplink arraying by using two $34 \mathrm{~m}$ beam waveguide antennas [3]. During the test, the two antennas launched synchronous remote carrier signal of $7.15 \mathrm{GHz}$ in orbit of Mars Global Surveyor spacecraft, shown in fig. 1. The MGS successfully received the synthesized transmitted signal. And synthesized signal quality and signal amplitude were recorded. The recording signals from telemetry datas to the ground receiving system were analyzed and enabled to maximize the received power of the spacecraft. The experiment verified that received signal power of spacecraft was $6 \mathrm{~dB}$ more than a single antenna when the two antennas had the same transmit power. 


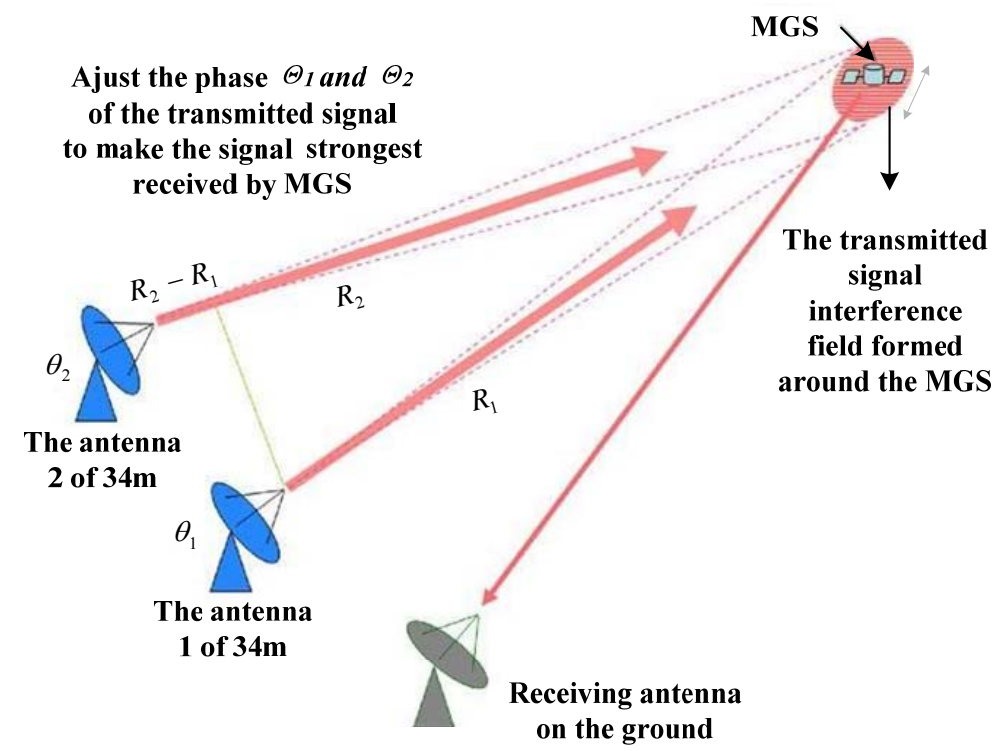

Fig. 1. The Uplink Arraying Principle Based on the Deep Space Probes MGS

In June 2008，V. Vilnrotter used deep space spacecraft EPOXI to carry out uplink antenna arraying signal synthesis experiments [4]. He used three $34 \mathrm{~m}$ antennas to track EPOXI and transmit remote control signals(one-way time delay of 2.9 minutes) in order to test whether arraying gain was $9.5 \mathrm{~dB}$ more than ever or not.

2007-2008, Larry D'Addario et al completed the uplink antenna arraying of small-scale and ground-based synthetic demonstration test [5]. Take advantage of five $1.2 \mathrm{~m}$ antennas whose frequency is between $14-14.5 \mathrm{GHz}$.

Each antenna has a $2 \mathrm{~W}$ power amplifier, so that all signals can be synthesized to generate $1 \mathrm{MW}$ of EIRP at the target. Successful trial showed that the loss of transmit signal which reached the target was less than $1 \mathrm{~dB}$. The phase stability of 1 hour, 24 hours and even 5-16 days could meet the requirements of synthesis.

At present, domestic research on the uplink antenna arraying is still in the stage of verification and simulation. Due to the study of uplink antenna arraying has just started in recent years, there are no proven cases and perfect synthesis algorithm in the domestic and international studies. By studying the spatial power synthesis performance of the uplink antenna arraying, analyze the performance of field intensity distribution, power synthesis, efficiency of multi-antenna and multi-beam at the target in space to determine the efficiency of the synthesis, the number of antennas and phase error in principle.

\section{The Theoretical analysis of spatial power synthesis technology}

\subsection{The Physical Background of Spatial Power Synthesis}

The multiplexed synthesis signal whose power is low is converted to required high-power radiation. Currently the power synthesis technology which is the most widely used is the circuit branch coupling synthesis technology and the spatial power synthesis technologies. The greatest advantage of spatial power synthesis technology is the synthetic efficiency of the system which is basically nothing to do with the number of solid-state devices. Therefore it is more suitable for the occasion which is full of more power output device [6].

The spatial power synthesis technology is a power synthesis method of microwave and millimeter wave which id proposed by the 1980s. The antenna arraying system is used by achieving the microwave power [7] [8]. Synthesis is divided into spatial power synthesis for a single device and antennas. The technology is used to reduce the power capacity requirement of a single device and a single antenna and cost of the project. widespread use of spatial power synthesis technology are multiple beam technology and phased array technology.

The Traditional spatial power synthesis was used for research and application of the single device [9][10]. Converting the antenna array into separate antennas for power synthesis is the uplink antenna 
arraying technology. In the space synthesis technique of multi-antenna signal, space pointing of each antenna which is based on the target position and the position of the antenna is calculated.

The calculation error of antennas will further result in the error of the transmitted signal of each antenna which is space-time delay difference at the target. The error results in the loss of a signal synthesis efficiency. The higher operating band, then the orbit accuracy requirements are increased accordingly.

Fig. 2 is far field power distribution diagram of Single and binary array antenna at different distances. The chart below shows the relationship between the synthesized signal power and the distance target error. Uplink arraying is mainly carried out for Deep Space Control. The purpose is to use a plurality of small diameter antenna to synthesize in the distant place of Deep Space Spacecraft and enhance the receiving power at the distant target.
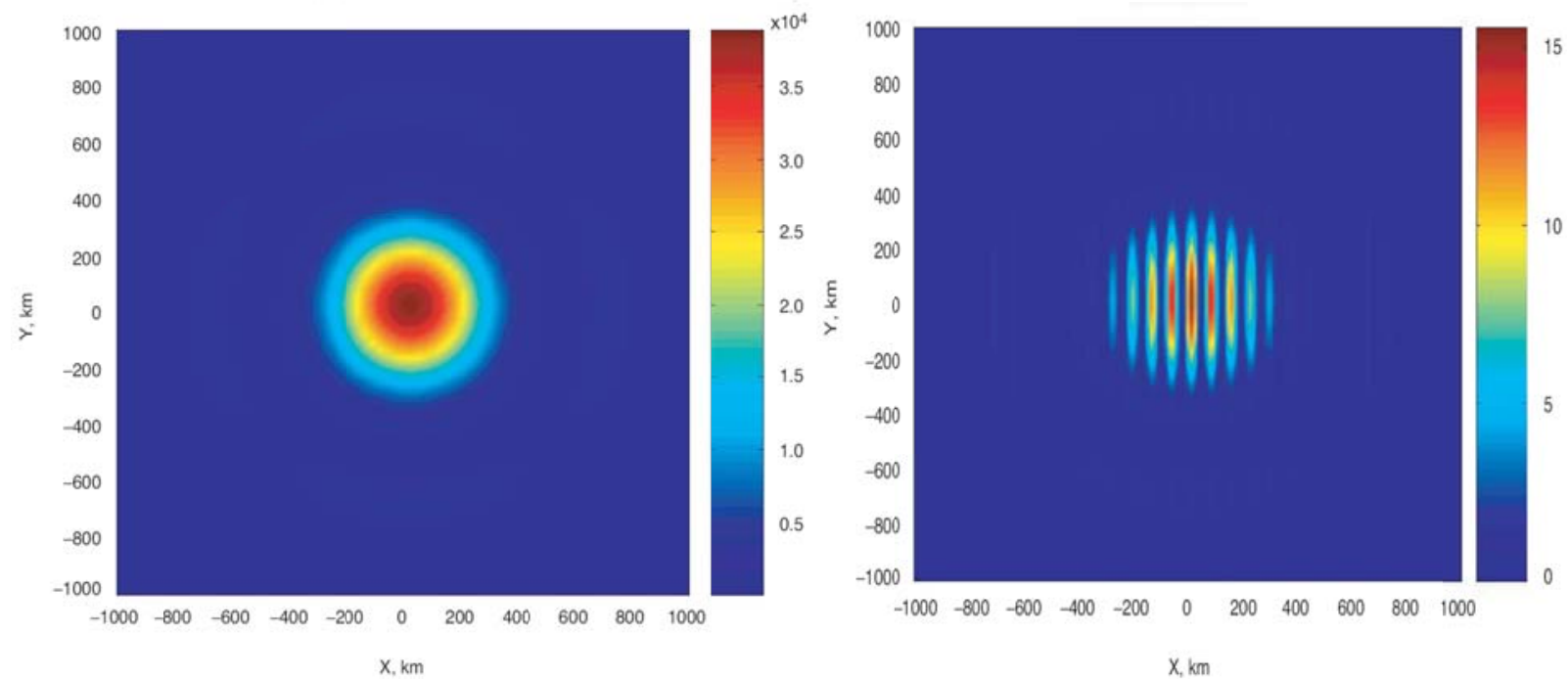

Fig. 2. The Spatial Power Synthesis Far-Field Pattern Based on the Uplink Antenna Arraying

\subsection{The Analysis of Spatial Power Synthesis Efficiency}

For example, derive the sapatial power synthesis efficiency in the phase error.

If the phase of each element could be consistent at the far field $P$, the synthesis efficiency would reach the maximum. However, in the actual process the phase of each antenna is difficult to remain consistent. The superimposed field strength of Binary Arrays in the far field as follows.

$E(t)=e_{1} \cos \left(\omega t+\phi_{1}\right)+e_{2} \cos \left(\omega t+\phi_{2}\right)$

In the formula, $\omega$ is the angular frequency, $e_{1}$ and $e_{2}$ are the amplitudes of Binary Arrays . $\phi_{1}$ and $\phi_{2}$ are the phases of Binary Arrays. Only consider the impact of the phase error here. Make $e_{1}=e_{2}$. The power density in the far field $P$ as follows.

$P=\bar{E}(t) / Z_{0}=2\left(e^{2} / Z_{0}\right)\left(1+\cos \left(\phi_{1}-\phi_{2}\right)\right)$

In the formula, $Z_{0}$ is the impedance of the free space wave. Make $\delta \phi=\phi_{1}-\phi_{2} .\left(0, \sigma^{2}\right)$ meets the normal distribution. $P_{\max }=4 e^{2} / Z_{0}$. The expectations of power density as follows $\left(\langle\cos x\rangle=\exp \left(-\sigma_{x}^{2}\right)\right.$, thereinto $\sigma_{x}$ is the standard deviation of $\mathrm{x}$ ).

$\bar{P}=2\left(\mathrm{e}^{2} / \mathrm{Z}_{0}\right)(1+\cos \delta \phi)=2\left(\mathrm{e}^{2} / \mathrm{Z}_{0}\right)\left(1+e^{-\sigma^{2} / 2}\right)$

So the power synthesis efficiency of Binary Arrays as follows.

$$
\eta=\bar{P} / P_{\max }=1 / 2\left(1+e^{-\sigma^{2} / 2}\right)
$$

Promote Binary Arrays to the $N$ element arrays whose power synthesis efficiency can be expressed as follows.

$$
\eta=\bar{P} / P_{\max }=\frac{1}{N^{2}} \sum_{k} \sum_{m} e^{-\sigma_{m m}^{2} / 2}
$$


In the formula, $k$ and $m$ is the number of antennas, $\sigma_{k m}^{2}$ is the variance which is the phase difference between antenna $k$ and antenna $m$ at the receiving end. When $k=m, \sigma_{k m}=0$. When each phase error is independent, it is subject to the normal distribution. When $k \neq m, \sigma_{k m}^{2}=2 \sigma_{0}^{2}$. The synthesis efficiency as follows.

$$
\eta=\bar{P} / P_{\max }=1 / N^{2} \sum_{k} \sum_{m \neq k} e^{-\sigma_{k m}{ }^{2} / 2}=\left(1-e^{-\sigma_{0}^{2}}\right) / N+e^{-\sigma_{0}^{2}}
$$

When $N \rightarrow \infty, \eta=\bar{P} / P_{\max }=e^{-\sigma_{0}^{2}}$.

Under the requirements of certain synthetic efficiency $\eta$, the relationship between the number $N$ of antennas and the phase error $\sigma$ as follows.

$$
\sigma=\sqrt{-\ln \frac{N \eta-1}{N-1}}
$$

\section{The Simulation of spatial power synthesis technology}

\subsection{The Relationship between the Phase Error and the Loss of Synthesis Gain under Each Antenna}

Consider the number of antennas sequentially selected as follows. $\mathrm{N}=2, \mathrm{~N}=4, \mathrm{~N}=6, \mathrm{~N}=8$ and $\mathrm{N}=10$. The relationship between the loss of synthesis gain and the root mean square phase error of each antenna as shown in fig. 3 .

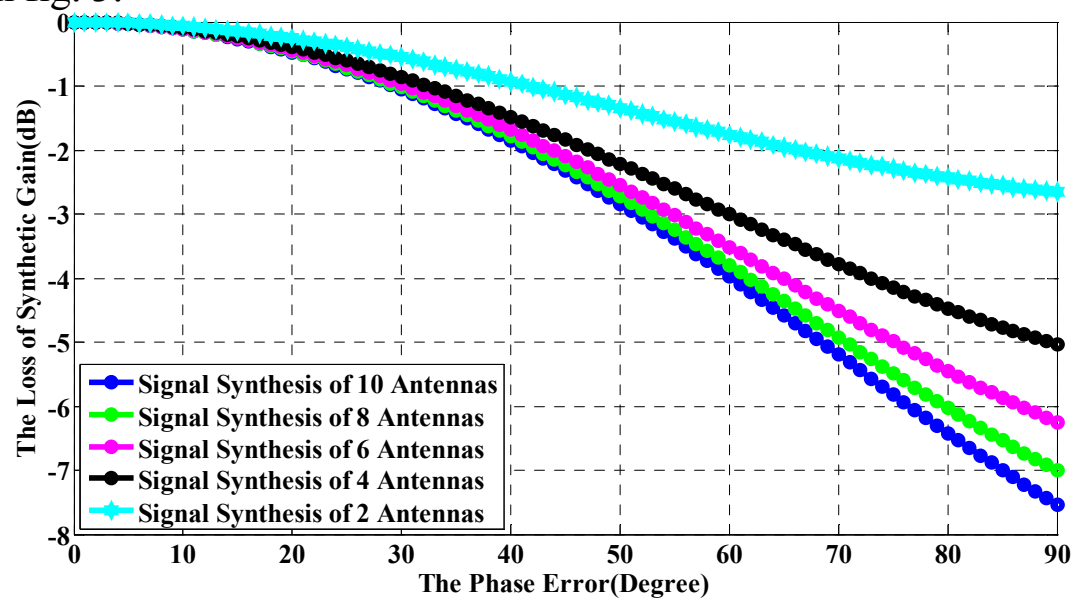

Fig. 3. The Relations between the Phase Error and the Loss of Synthetic Gain under Different Antennas

With the numbers of antennas increasing, the loss of synthesis gain will increase under each phase error. In addition, as the number of antennas increasing, the the increasing degree of the loss of synthesis gain will be less when phase error is increasing. Since the loss of synthesis gain is very close within phase error $30^{\circ}$, the corresponding loss of power synthesis gain at different numbers of antennas under different phase error is set out as shown in Table 1. From the data in the table, the maximum of the loss of synthesis gain is less than $3 \mathrm{~dB}$ when the number of antennas is two. When the number of antennas is ten, the phase error is $80^{\circ}$ the loss of synthesis gain is $-6.42 \mathrm{~dB}$. Therefore determine how to select the numbers of antennas in accordance with prescribed limit of the loss of synthesis gain.

\subsection{The Relationship between the Number of Antennas and the Power Synthesis Efficiency}

When the phase error is respectively $40^{\circ}, 50^{\circ}, 60^{\circ}, 70^{\circ}$ and $80^{\circ}$, the relations between the number of antennas and power synthesis efficiency as shown in fig. 4. 


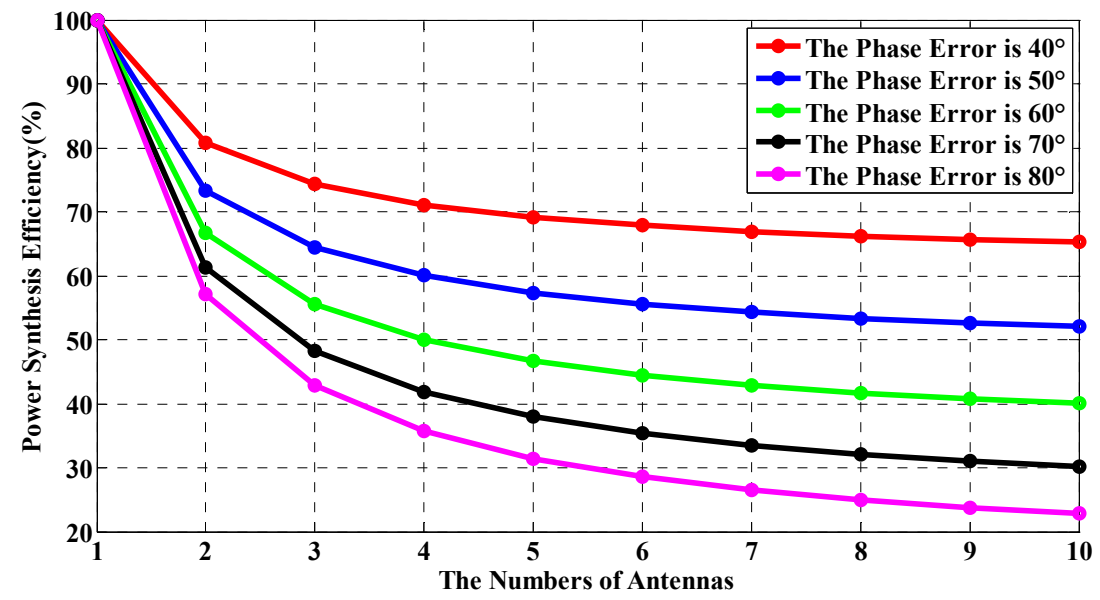

Fig. 4. The Relations between the Numbers of Antennas and Power Synthesis Efficiency under Different Phase Error

The figure shows that the less the number of antennas, the higher the synthesis efficiency. The higher the phase error, the lower the synthesis efficiency. When the number of antennas is two, the corresponding difference of the synthesis efficiency between the phase error $40^{\circ}$ and $80^{\circ}$ is about $23 \%$. When the numbers of antennas are more than five, the corresponding difference of the synthesis efficiency between the phase error $40^{\circ}$ and $80^{\circ}$ is at least $40 \%$.

Record the power synthesis efficiency corresponding to the numbers of antennas as shown in table 2. From the table 2, the power synthesis efficiency corresponding to each phase error showed a decreasing trend with the numbers of antennas increasing. When the numbers of antennas are ten, the power synthesis efficiency corresponding to the phase error $80^{\circ}$ is 0.23 . When the numbers of antennas are two, the power synthesis efficiency corresponding to the phase error $40^{\circ}$ is 0.81 .

Table 1. The Relations between the Numbers of Antennas and the Loss of Power Synthesis Gains

\begin{tabular}{|c|c|c|c|c|c|}
\hline $\begin{array}{c}\text { Loss } \\
\text { of } \\
\text { Synthesis Phase } \\
\text { The }_{\text {Numbers }}^{\text {Gain }} \text { of (dB) }\end{array}$ & $40^{\circ}$ & $50^{\circ}$ & $60^{\circ}$ & $70^{\circ}$ & $80^{\circ}$ \\
\hline Antennas $_{2}$ & & & & & \\
\hline 4 & -0.93 & -1.35 & -1.76 & -2.13 & -2.43 \\
\hline 6 & -1.48 & -2.21 & -3.01 & -3.78 & -4.48 \\
\hline 8 & -1.68 & -2.55 & -3.52 & -4.51 & -5.45 \\
\hline 10 & -1.79 & -2.73 & -3.80 & -4.93 & -6.03 \\
\hline & -1.85 & -2.84 & -3.97 & -5.20 & -6.42 \\
\hline
\end{tabular}


Table 2. The Relations between the Numbers of Antennas and the Power Synthesis Efficiency

\begin{tabular}{|c|c|c|c|c|c|}
\hline $\begin{array}{l}\text { Power The } \\
\text { Synthesis Numbers } \\
\text { Efficiency Antenna }\end{array}$ & 2 & 4 & 6 & 8 & 10 \\
\hline $40^{\circ}$ & 0.81 & 0.71 & 0.68 & 0.66 & 0.65 \\
\hline $50^{\circ}$ & 0.73 & 0.60 & 0.56 & 0.53 & 0.52 \\
\hline $60^{\circ}$ & 0.67 & 0.50 & 0.45 & 0.42 & 0.40 \\
\hline $70^{\circ}$ & 0.61 & 0.42 & 0.35 & 0.32 & 0.30 \\
\hline $80^{\circ}$ & 0.57 & 0.36 & 0.29 & 0.25 & 0.23 \\
\hline
\end{tabular}

3.3 The Relationship between the Phase Error and the Synthesis Efficiency under the Synthesis of Each Antenna

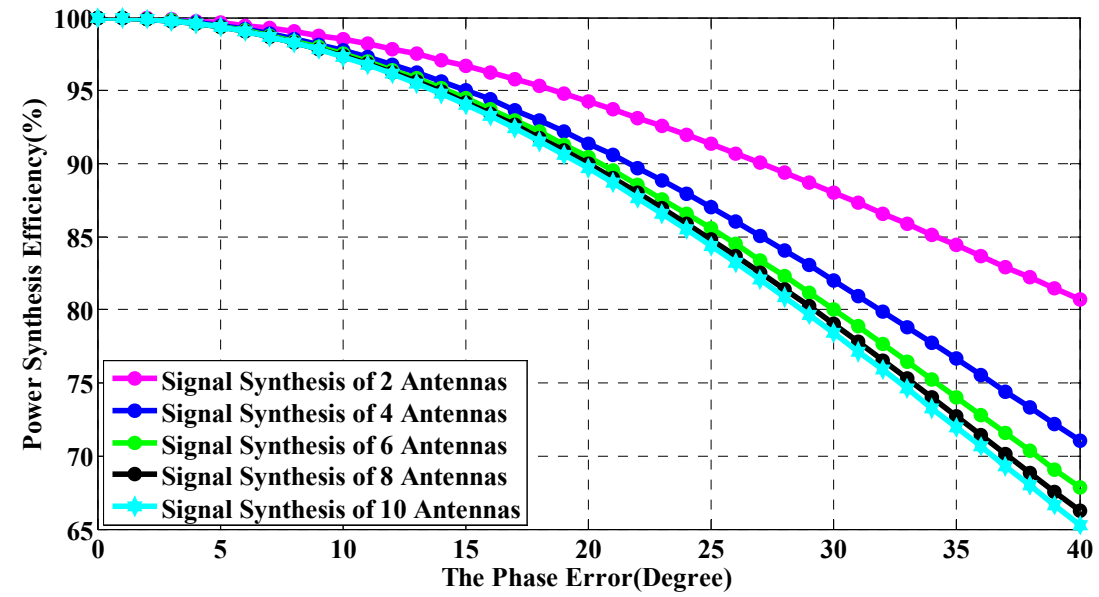

Fig. 5. The Relations between the Phase Error and the Synthesis Efficiency under Signal Synthesis of Different Antennas

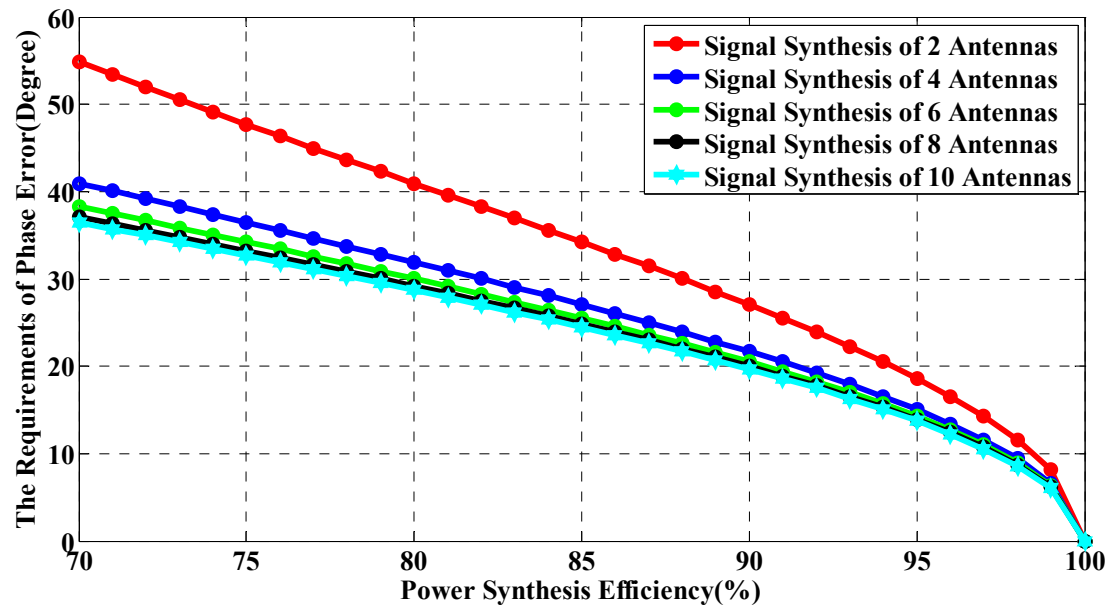

Fig. 6. The Requirements of the Synthesis Efficiency for Phase Error under Signal Synthesis of Different Antennas

When the numbers of selected antennas are $\mathrm{N}=2, \mathrm{~N}=4, \mathrm{~N}=6, \mathrm{~N}=8$ and $\mathrm{N}=10$, the relationship between the synthesis efficiency and rms of the phase error for each antenna is respectively shown in fig. 5 and fig. 6 . As the figure shows, the antennas synthesis efficiency would show a decreasing trend 
with the phase error increasing. Similarly the requirements of the phase error would decrease with the antennas synthesis efficiency increasing. When the phase error is within $10^{\circ}$, the synthesis efficiency corresponding to each antenna is not very different. When the phase error is $40^{\circ}$, the synthesis efficiency corresponding to ten antennas is $65 \%$. At the same time the synthesis efficiency corresponding to two antennas is $80 \%$. Meanwhile the requirements of the phase error would be lower if the requirements of the synthesis efficiency were not high. When the synthesis efficiency is $70 \%$, the phase error corresponding to two antennas is $55^{\circ}$.

3.4 The Relationship between the Number of Antennas and the Phase error under Different Synthesis Efficiency

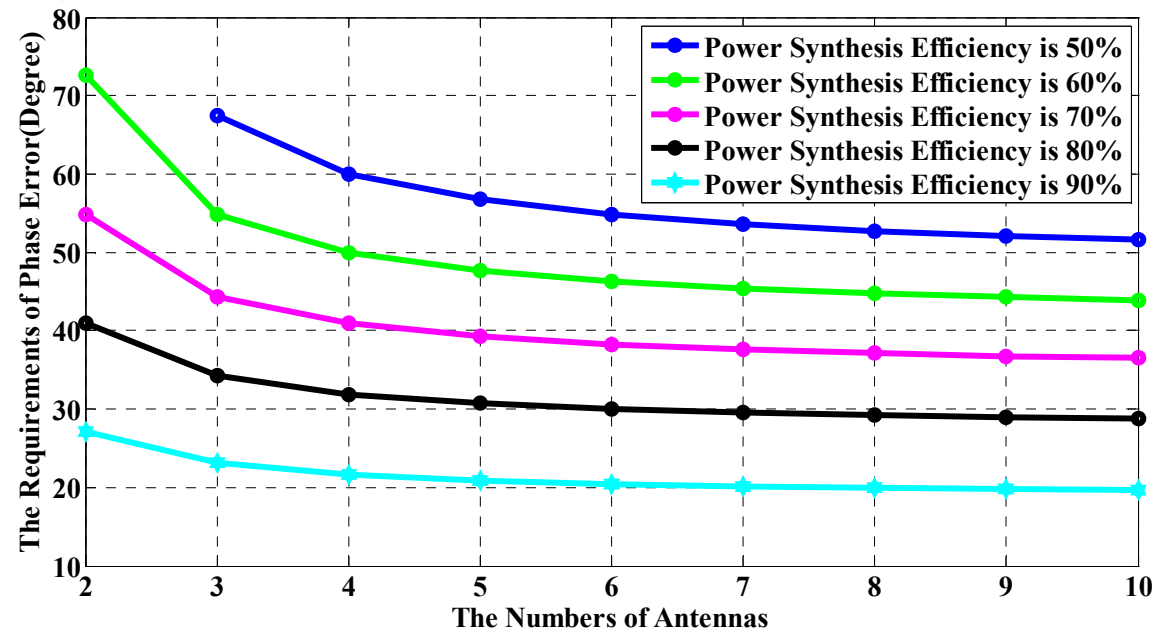

Fig. 7. The Numbers of Antennas on the Requirement of the Phase Error under Different Power Synthesis Efficiency

When synthesis efficiency is respectively $50 \%, 60 \%, 70 \%, 80 \%$ and $90 \%$, the requirements of the phase error corresponding to the numbers of antennas under different power synthesis efficiency are shown in fig. 7. The figure shows that the requirements of phase error are more demanding with the increasing numbers of antennas under the same requirements of synthesis efficiency. When the numbers of antennas are higher, the requirements of the phase error would tend to be a constant value. According to the relations between the numbers of antennas and the phase error, the value of the phase error is set to be an invalid value when the synthesis efficiency is $50 \%$. The phase error remains substantially constant corresponding to different synthesis efficiency when the numbers of antennas are selected to be four. Meanwhile, the difference of the phase error corresponding to the adjacent synthesis efficiency is relatively close when the numbers of antennas are fixed.

\section{Conclusions}

From the above analysis, the space synthesis efficiency is associated with the magnitude of each element, the number of antennas and the phase error. The more the numbers of antennas, the higher the phase error, the more the loss of synthesis gain, and the lower the corresponding power synthesis efficiency. In addition, the error of position, error of time-delay and error of element spacing are all affected by the phase error at the far field.

Under the optimal circumstances of the relevant phase, the power $P_{\text {sum }}$ of the synthesis signal is the power $P_{e}$ of a single transmitter multiplied by the quadratic times of number of array elements $N$ which is $P_{\text {sum }}=N^{2} P_{e}$. However it's not necessarily related to a system of the uplink Antenna Arraying. In the case of moderate correlation, the relative phase stability is easier to achieve. Therefore, consider the aperture of antennas, the spacing of antennas, the level of signals and the cost of arraying which are actually possible to achieve. Look for the best point of synthesis to determine reasonable requirements of synthesis efficiency. 


\section{References}

[1] Davarian F. Uplink Arrays for the Deep Space Network[J]. Proceedings of the IEEE, 2007, 95(10): 1923-1930.

[2] Rogstd D H, Mileant A, Pham T T. Antenna Arraying Techniques in the Deep Space Network[M]. New York: Wiley InterScience, 2003.

[3] V. Vilnrotter, et al. Uplink Arraying Experiment with the Mars Global Surveyor Spacecraft[R], JPL IPN Progress Report 42-166F, 2006.

[4] V. Vilnrotter, P. Tsao, et al. EPOXI Uplink Array Experiment of June 27, 2008[R], JPL IPN Progress Report 42-174E, 2008.

[5] Addario L D, Proctor R, Trenh J, et al. Uplink Array Demonstration with Ground-Based Calibration[R]. Jet Propulsion Laboratory, Pasadena, California: The Interplanetary Network Progress Report 42-176. February 15, 2009: 1-69.

[6] Durkin M F, Eckstein R J, Mills M D. 35 GHz Active Aperture. Microwave Symposium Digest[R]. MTT-S International. 1981, 81(1): 425-427.

[7] K. A. Hummer, K. Chang. Spatial Power Combining Using Active Microstrip Patch Antennas[J]. Microwave and Optical Technology Letters. 1988, 1(1): 8-9.

[8] Jiang Xin, Ortiz Sean,Mortazawi Amir. A Novel Ka-band 1 to 8 Power Divider/ Combiner[C]. Mierowave Symposium Digest, 2001 IEEE MTT-S International. 2001, 1(5): 20-25.

[9] DeLisio M.P, York R.A. Quasi-optical and spatial power combining [J]. IEEE Transactions on Microwave Theory and Techniques, 2002, 50(3): 929-936.

[10]Hummer K. A, Chang K. Spatial power combining using active microstrip patch antennas[J]. Microwave and Optical Tech.Lett, 1988, 1(1): 8-9. 